\title{
Advances in the Role of ADF / Cofilin in Central Nervous System
}

\author{
Ke Li ${ }^{a}$, Xiaoxin Wang ${ }^{b}$,Weidong $\mathrm{Li}^{\mathrm{c}}$,Jiali Cheng ${ }^{\mathrm{d}}$, Yanhang Zhang ${ }^{\mathrm{e}}$ and Lijuan \\ $\mathrm{Hou}^{\dagger}$
}

PE and Sports Science Department, Beijing Normal University, Beijing, 100875, China

a18865382787@163.com, b201521070030@bnu,edu,cn, c463907772@qq.com

d405270197@qq.com, e302590711@qq.com, fhoulj@bnu.edu.cn

\begin{abstract}
Keywords: ADF/cofilin; Central Nervous System; Actin; Cytoskeleton; Exercise
Abstract. Extracellular signaling regulates cell motility, growth, differentiation, gene expression, polarization and death. Activation of Actin cytoskeleton is an important condition for the occurrence of these cellular responses and morphological changes. The ADF / Cofilin family of proteins is an important regulator of Actin cytoskeleton regulation, recent studies have shown that changes in the content and activity of these proteins are associated with a variety of neurological diseases such as Parkinson's disease, Alzheimer's disease etc. Exercise can change the activity of the family protein and help to delay or cure the disease. This article reviews the role of ADF / Cofilin family proteins in the nervous system in order to provide new ideas for future disease treatment.
\end{abstract}

\section{Introduction}

The Actin cytoskeleton is formed by the combination of fiber bundles and Actin filaments. In the Actin composition, there is a spherical monomer Actin (G-Actin) and filamentous multimeric Actin (F-Actin), the dynamic change between the two can make Actin play good and stable biological and physiological functions, that is, Actin remodeling (Actin remodeling) phenomenon. Actin depolymerization factor ADF / cofilin family of proteins is a class of proteins that are highly bound to Actin, which are commonly found in eukaryotic cells other than mature erythrocytes and spermatids. They play an important role in Actin skeleton remodeling.

In recent years, research on the relationship between control of ADF/cofilin with learning and memory function and other related advanced and neural system disease, are also increasingly in-depth. Therefore, by summarizing the research progress of the role of ADF/cofilin in the central nervous system, we can provide a new idea for the nervous system diseases.

\section{Mechanism of ADF/Cofilin Family Proteins Regulating Actin Dynamics}

In most vertebrates, especially in all mammals, the ADF / cofilin family of proteins includes ADF, as well as non-myofibro-cleavidin (n-cofilin), which is expressed primarily in the brain and liver, and more in muscle expression of muscle type cofilin (m-cofilin) [1].The main function of cofilin is to cleave the filaments on Actin, especially for the filamentous structure of F-Actin.

Roland et al. [2] demonstrated that ADF / cofilin exerts depolymerization activity by cleaving Actin filaments and enhancing the rate of Actin monomer leaving the end of Actin filaments. Studies have shown that ADF / cofilin expression controls the polar motility of cells[3,4]. This makes ADF / cofilin indispensable in cellular activity such as cell migration, cell proliferation, cytoplasmic circulation, cytoplasmic cleavage, and so on.

\section{Regulation of ADF/Cofilin Family Protein Activity}

ADF / cofilin family protein activity is regulated by autophosphorylation and dephosphorylation. Ser3 site phosphorylation inhibits the depolymerization activity of ADF / cofilin. LIM kinase and TES kinase on the ADF / cofilin Ser3 site for specific phosphorylation, the ADF / cofilin loss of depolymerization activity, causing Actin remodeling.Limk family, including limk1 and limk2, in the neuronal dendritic spine limk1 content is very rich, limk2 can also phosphorylate cofilin to inactivate, 
inducing neuronal differentiation. Whereas TESK family protein kinases are mainly expressed in the reproductive system [5]. But its activity was restored after dephosphorylation of filamentous phosphatase (SLingshot, SSH) and CIN [6]. SSH is widely distributed in various tissues, CIN phosphatase is an important regulator of cofilin phosphatase [7] in the central nervous system, regulating the local activity of cofilin.

That is to say, the biological activity of cofilin plays an important role in "opening and closing" to regulate the depolymerization and polymerization of Actin and play a variety of physiological functions [8-9].

\section{Function of ADF/Cofilin Family Proteins in Central Nervous System}

ADF/Cofilin Family Proteins and Synaptic Plasticity of Dendritic spines. Actin cytoskeleton plays an important role in post-synaptic structure and function, as well as synaptic plasticity changes [10]. The synaptic plasticity of the dendritic crest is based on the Actin network and the high activity of the ADF / cofilin leads to a smaller dendritic crest and the formation of immature cristae [11]. Cofilin has become a key regulator of Actin. By cutting Actin filaments, it can dissolve old Actin fibers, aggregate and transform new dendritic spines, it is closely related to neuronal synaptic plasticity, and human learning and memory function [12].

The activity of Actin is often related to the size of dendritic spines, and the increase in cofilin activity leads to the formation of small dendritic spines and immature naive spinous processes, but in some cases cofilin can also promote synaptic enhancement [13]. Such as in the development of the hippocampus, many factors can regulate the cofilin ser3 binding site phosphorylation status to regulate the migration of neurons, the establishment of fiber contact, cerebral cortex cell cycle changes and the formation of synaptic processes [14]. In addition to inducing synaptic loss, combined with electrophysiological detection also found that can damage to synaptic plasticity, including basic synaptic transmission and LTP formation [15].

It can be seen cofilin as the central nervous system important Actin depolymer, cofilin-Actin polymerization may be associated with neurodegenerative diseases and brain aging.

ADF/Cofilin Family Proteins and Parkinson Disease . In Parkinson's disease (PD) animal model or the patient's brain, the major pathological changes occurred in the substantia nigra were dopaminergic neuron loss and to the striatum projection of the medium-sized multi-spine neurons dendritic spine reduction [16-17]. A recent study has shown that leucinerich repeat kinase2 (LRRK2) is involved in the pathogenesis of PD. LRRK2 is mainly expressed in the brain striatal projection neurons (SPNs). The knockdown of the LRRK2 gene results in a decrease in protein kinase A (PKA) activity at SPNs, the expression of postsynaptic density protein (PSD-95) decreased, cofilin was over-phosphorylated, dendritic spine density decreased, and filamentous dendritic spines were more than mushroom-like dendritic spines, developmental delay, showing a significant pathological features of PD.(Fig. 1) [18].LRRK2 only through cofilin to control the development of dendritic spines. Suggesting that the researchers may be able to signal the LRRK2-PKA-Cofilin-Transduction pathway to find a therapeutic target for the treatment of PD [18].

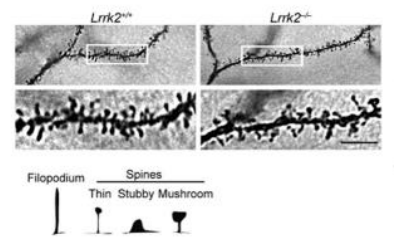

Fig. 1, Golgi-Cox staining of SPNs from P15 littermate $L R R K 2+/+$ and $L R R K 2-/-$ pups. The bottom panels are enlargement of boxed areas of the upper panels. Scale bar: $5 \mu \mathrm{m}$. The inset shows schematic representation of filopodium and different types of dendritic spines. Cited from reference [18].

ADF / Cofilin Family of Proteins with Aging. The activity of Rho GTPases affects the function of downstream cofilin [19]. The Rho GTPases family mainly includes Rho, Rac and Cda42, Rho includes RhoA, RhoB and RhoC three isomers. Activation of RhoA pathway, F-Actin rapid 
polymerization, mediated growth cone collapse, protrusion retraction, inhibition of axon extension; and Rac, Cdc42 has opposite role [20].

The expression of RhoA in the cortex of the aged rats was significantly higher than that of the young rats, while the expression of Rac1 and Cdc42 in the RhoA group was not significantly changed in the old group. There was a significant cofilin aggregation in the aged rat cortex, and the synaptic density was significantly decreased, indicating that the cofilin aggregation accompanied by brain aging was one of the causes of synaptic loss. The 12-week regular exercise could significantly reduce the senile cortex cofilin aggregation, which in turn increases the cortical synaptic density [21].

ADF / Cofilin Family of Proteins with Alzheimer's Disease. Unlocking if cofilin1's inactive is a cause or consequence of $\mathrm{AD}$ pathology requiring further investigation [22]. We found that both in vivo and in vitro a significant increase in the ratio of pcofilin 1 / cofilin1 indicates that cofilin1 is inactivated in the brain of AD patients and APP / PS1 mice as a result of AD pathology(Fig. 2). Studies have shown a significant increase in the proportion of pcofilin 1 / cofilin1 in the brain of APP / PS1 mice compared with the same litter WT control, suggesting that AD pathology can accelerate the aging process by cofilin 1 phosphorylation. These changes are mainly related to SSH1 inactivation. Experiments show that $\gamma$-secretase activity can inhibit the SSH1 and then induced cofilin1 phosphorylation [23].
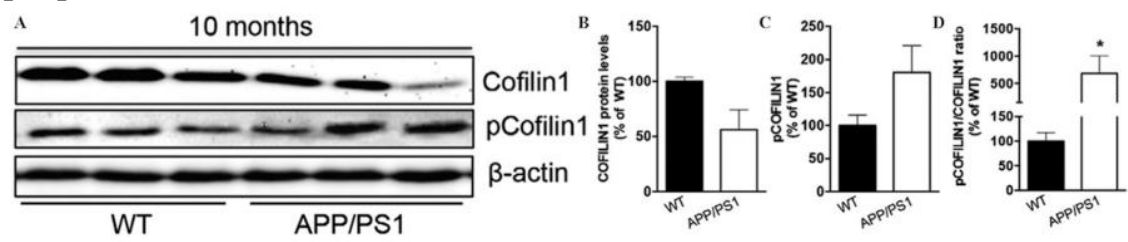

Fig. 2, Alzheimer's disease pathology-associated changes of the Cofilin1 pathway proteins in the brain of APP/PS1 mice. Cofilin1 and pCofilin1 protein levels were respectively reduced by $\sim 45 \%$ (Fig. 2A) and increased by $\sim 80 \%$ (Fig. 2B) in AD mice, with respect to the littermate matched WT controls. Cited from reference [23].

\section{Effects of Exercise on ADF/Cofilin Family Proteins}

The results of the study by Ana F.B. Ferreira et al. show that moderate exercise regulates synaptic and structural proteins in the brain regions of the brain, which may play an important role in motor induced brain plasticity [24]. Gu et al showed aerobic exercise can improve the June age APP+PS1 double transgenic mice's ability of learning and memory and behavior, reduce the frontal and temporal lobe A $\beta$ deposition, reduce the abnormal aggregation of cofilin, thus increasing the cortical Syn content, increase in synaptic density, is one of the mechanisms of movement delay in patients with $\mathrm{AD}[25]$. Zhao Li et al. study results suggest that moderate exercise can induce the overall biological fitness, and movement by regulating the Rho family of GTPase enzyme activity in the cortex and excessive accumulation of inhibition of cortical cofilin, delayed the loss caused by the aging of cortical synapses [21].

\section{Conclusion}

The ADF / Coffin family of proteins is an important regulator of Actin cytoskeleton, they not only participate in, but also in various neurological diseases such as Parkinson's disease, which plays an important role in the pathogenesis of Alzheimer's disease, through the movement can delay the loss of synaptic activity or change the content of the protein family, to protect the nervous system.

In the future, we will be able to gain a better understanding of the pathogenesis and the protective effects of the family proteins in the nervous system, we also can improve the function of the central nervous system by exercise. 


\section{Acknowledgements}

This study has been supported by the National Natural Science Foundation of China (31401018). National Training Programs of Innovation and Entrepreneurship for Undergraduates in 2016 (201610027008). Associate Professor Lijuan Hou is the corresponding author of this paper and her research interests include motor physiology and motor control. E-mail is houlj@bnu.edu.cn.

\section{References}

[1] B. J. Pope, K. M. Zierler-Gould and R. Kuhne et al. Journal Biol Chem. Vol. 279 (2004), p. 4840-4848.

[2] J. Roland, J. Berro and A. Michelot et al. Biophys. Vol. 94 (2008), p. 2082-94.

[3] P. D. Sarmiere, J. R. Bamburg. Neurobiol. Vol. 58 (2004), p.103-17.

[4] C. G. Dos Remedios and D. Chhabra et al. Physiol Rev. Vol. 83 (2003), p. 433-473.

[5] J. Tastet, P. Vourc'h and F. Laumonnier, et al. Biochem Biophys Res commun. Vol. 420 (2012), p. 247-252.

[6] B. W.Bernstein and J. R. Bamburg. Trends Cell Biol. Vol. 20 (2010), p. 1878-195.

[7] A. Gohla, J, Birkenfeld and G. M. Bokoch. Nat Cell Biol. Vol. 7 (2005), p. 21-29.

[8] N. V. Oleinik and S. A. Krupenko. Oncogene. Vol. 29 (2010), p. 6233-6244.

[9] L. Zhang, J. Luo and J. P. Wan. et al. Development. Vol. 138 (2011), p. 455-464.

[10] P. Hotulainen, C. C. Hoogenraad. J cell Biol. Vol. 189 (2010), p. 619-629.

[11] J. Gu, C. W. Lee and D. Komlos, et al. Nat Neurosci. Vol. 10 (2010), p.1208-15.

[12] M. B. Rust, C. B. Gurniak and M. Renner. et al. EMBO J. Vol. 29 (2010), p. 1889-1902.

[13] J. Gu, C. W. Lee and Y. Fan. et al. Nat Neurosci. Vol. 13 (2010), p. 1208-1215.

[14] T. Y. Huang, C. DerMardirossian and G. M. Bokoch. Curr Opin Cell Biol. Vol. 18 (2006), p. 26-313.

[15] C. C. J, C. Sun and B. Chen. et al.J. Biol Chem. Vol. 287 (2012), p. 3919-3929.

[16] I. S. Pienaar, D. Burn and C. Morris. et al. Mol Neurobiol. Vol. 45 (2012), p. 126-143.

[17] R. M. Villalba and Y. Smith. Front Neuroanat. Vol. 4(2010), p. 133.

[18] L. Parisiadou, J. Yu and C. Sgobio. et al. Nat Neurosci. Vol. 17 (2014), p. 367-376.

[19] Q. Deng, W. Cai and S. Li. et al. Brain Res Bull. Vol. 99(2013), p. 140-144.

[20] M. J. Williams. Small GTPases. Vol. 3 (2012), p. 1.

[21] L. Zhao, Y. Li and B. Y. Gu. Journal of Beijing Sport University. Vol. 36 (2013), p. 61-69.

[22] F. X. Guix, T. Wahle, K. Vennekens, A. Snellinx, L. Chavez-Gutierrez, G. Ill-Raga, E. Ramos-Fernandez, C. Guardia-Laguarta, A. Lleo, M. Arimon, O. Berezovska, F.J. Munoz, C.G. Dotti and B. De Strooper. EMBO. Vol. 36 (2013), p. 660-673.

[23] Eugenio Barone, Sebastien Mosser, C. Patrick, Fraering. Biochimica et Biophysica Acta. Vol. 1842 (2014), p. 2500-2509.

[24] F. B. Ana, Ferreira, C. Caroline, Real, C. Alice, Rodrigues, S. Adilson, Alves, R. G. Luiz, Britto. Brain research. Vol. 1361(2010), p. 31-42. 
[25] B. Y. Gu, X. W. Yu, Y. Li and L. Zhao. Journal of Beijing Sport University. Vol. 38(2015), p. 77-82. 